\title{
Application of Magnetically Controlled Sorbents for Detoxication
}

\author{
O.A. Butusova \\ Moscow Aviation Institute (National Research University), Volokolamskoe shosse, 4, 125993, Moscow, Russia \\ gxtl@mail.ru
}

Article History: Received: 11 January 2021; Accepted: 27 February 2021; Published online: 5 April 2021 Abstract: This work shows the conditions that ensure the efficiency of the device for extracorporeal
detoxification using new technologies using magnetically controlled sorbents. The above technique allows
calculating and, accordingly, optimizing the parameters of one of the important components of this device - the
unit for sorption and removal of toxins from biofluid. A weighted estimate of the required concentration of
magnetically controlled sorbents in the biofluid being purified, the amount and concentration of an emulsion
containing a magnetically controlled sorbent, and the duration of the procedure ensures the safety and
effectiveness of this method of medical care.

Keywords: Magnetically controlled sorbents, adsorption, toxins, biofluids.

\section{Introduction}

The principle of using magnetically controlled sorbents in detoxification processes consists in introducing them into the environment to be cleaned (blood, plasma, lymph) and absorbing (sorption) of harmful impurities contained in biofluid on the developed surface of magnetically controlled sorbent granules. Then, using a magnetic field, the waste particles are separated and removed together with harmful impurities from the biofluid bed. The effectiveness of the sorbent depends on its particles size [1-6]. For granulated sorbent, the capacity can be very high due to the nano- and microdisperse structure of the suspension of magnetically controlled sorbents: the size of the granules can vary from $50-60 \mathrm{~nm}$ to several microns.

Accordingly, the specific (per unit mass of particles) surface area of magnetically controlled sorbents can reach several thousand $\mathrm{m}^{2}$. A layer of an active sorbent, for example, activated carbon, is applied to the surface of each granule. Taking into account the small size of the particles themselves of magnetically controlled sorbents, the thickness of its adsorbing shell (layer of activated carbon) is negligible - in the limit it can be a monolayer of carbon atoms. As is known, the rates of the establishment of adsorption-desorption equilibrium in carbon coatings of small thickness are significantly increased, which provides a high speed of the specified method. The same effects were applied for creation and modelling of composite materials of various nature [7-21].

It should be noted that the total area of the adsorbing surface is so large that desorption practically does not reduce the surface density of the reagent. For magnetically controlled sorbents with small particles covered with thin carbon shells, the equilibrium concentration of the adsorbate in the near-surface layers of the sorbent is established very quickly. Therefore, with a sufficient concentration of magnetically controlled sorbents in the biofluid being purified, a decrease in the concentration of toxins to preset values is achieved almost instantly.

Hence, it is clear that the concentration of the reagent in the biofluid does not depend on time. It installs almost instantly and remains unchanged. In addition, the concentration of the reagent does not depend on the amount of the sorbent used, because usually there is a lot of it, taking into account the specific surface area of more than $100 \mathrm{~m}^{2} / \mathrm{g}$. This facilitates its dosing, for example, in order to ensure the required concentrations of solutions or medical dosage rates. At the same time, the biofluid processing process can be carried out in real time, it becomes cheaper, its productivity and environmental friendliness increase.

The choice of the type of sorbent material is based on the analysis of its physicochemical characteristics, taking into account the functional task assigned to it.

It should be noted that among many known types of sorbents, the use of activated carbon as a sorbent shell, taking into account the extremely developed pore surface, which, moreover, is easily accessible, seems to be quite promising.

\section{Evaluation of the parameters of the detoxification system}

For convenience, we present a typical graph of the Langmuir adsorption isotherm in the form of two graphs, using a scale $C_{a}$ with two scales, as shown in Fig. 1, where curve 1 with a lower scale and curve 2 with an upper scale represent, respectively, the initial and final parts of the adsorption isotherm in the form $\Gamma / \Gamma_{\max }=\theta\left(C_{a}\right)$. The values $\Gamma_{\text {and }} \Gamma_{\max }$, which determine the current amount of adsorbed substance and the maximum amount of adsorbed substance by the surface of the sorbent, are determined empirically by known methods[22-31].

It is clear that the lower curve in Fig. 1 corresponds to a solution with the concentration of the dissolved (adsorbed) component in the segment $[0,300]$. Then the curve becomes so flat that it is more convenient to trace its change with a significant compression of the scale along the abscissa axis (300 times). 


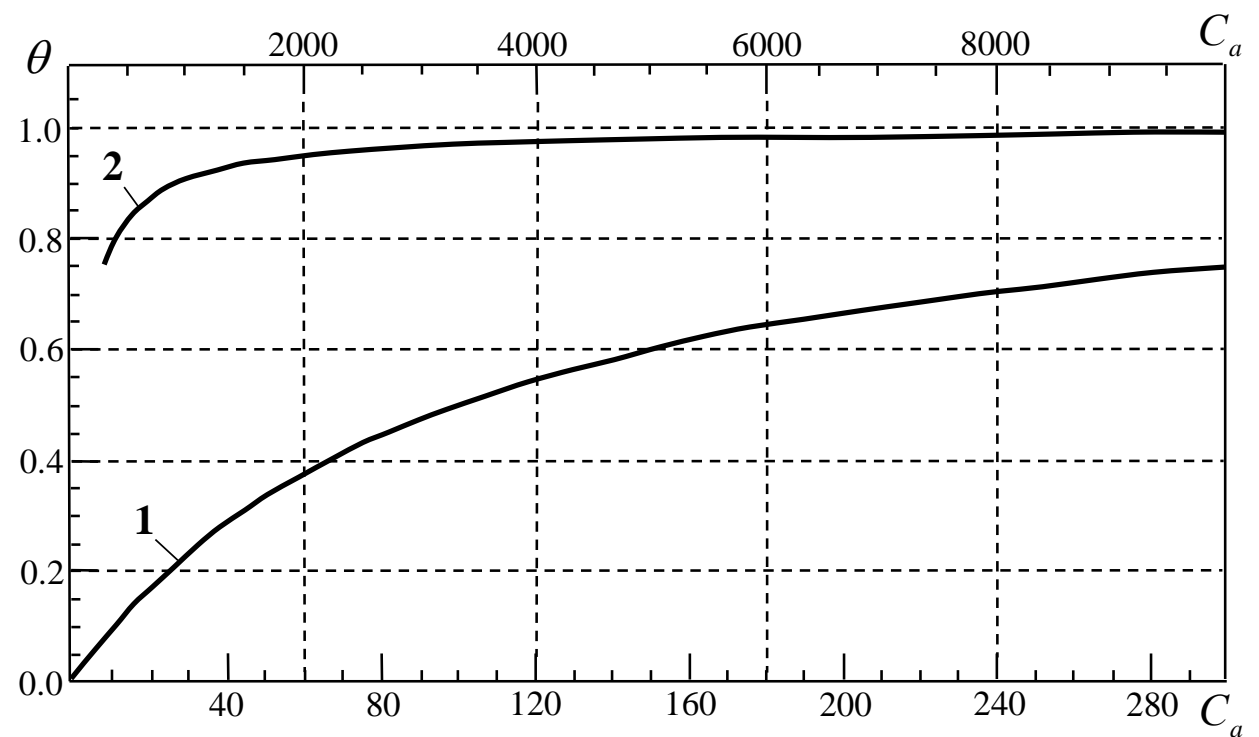

Figure: 1. Typical dependence of the relative filling of the adsorbent surface $\theta=\Gamma / \Gamma_{\max }$ on the adsorbate concentration $C_{a}$ in the biofluid being purified.

Fig. 1shows, that in the initial section, the concentration of the dissolved adsorbate $C_{a}$ is almost linearly related to the relative density of its distribution on the surface of the sorbent $\theta$. With the transition to the saturation region, the rate of filling the sorbent surface significantly decreases and the sorbent loses its former efficiency. Therefore, it is advisable to choose the operating mode of the sorbent in the linear section of its operation, for example, when the density coefficient is $\theta<0.4$. The equilibrium state in this process is very similar to heat transfer regularities described in [32-41].

To assess the main parameters of the extracorporeal detoxification system using this sorbent, let us take a unit volume of biofluid with a toxin, for which the MPC level, for example ( $C_{M P C}=30$ conv. units)

Along the curve in Fig. 1 we find that the indicated concentration $\mathrm{Ca}=\mathrm{C}_{\mathrm{MPC}}$ corresponds to the value $\theta=0.25$ on the part of the curve, which, as noted above, will ensure the efficiency of the sorbent. The reciprocal of this value $\theta$ determines the required multiplicity of the reserve of the sorbent surface occupied by toxin molecules in relation to the total sorbent surface. The required sorption surface area increases with an increase in the molecular weight of the adsorbate.

It is known that complex organic compounds usually have a bulky structure and their spatial configuration is not reduced to linear chains. Therefore, for simplicity, we will consider the adsorbate (toxin) molecules of a spherical shape with a molecular weight $m$ (earlier $M_{t}$ ), a radius of $r$ and a diametrical cross-sectional area $A=\pi r^{2}$ (earlier $S_{d}=\pi r^{2}$ ). Particles of a magnetically controlled sorbent will also be considered spherical with a radius $R$ and a surface area $S=4 \pi R^{2}$ (previously $\left.S_{M Y C}=4 \pi R^{2}\right)$

Assuming $R \gg r$, let us consider the case of the placement of adsorbate molecules on the surface. It is known that complex organic compounds usually have a bulky structure (they cannot be reduced to simple linear chains). As an example, consider the case of placement of spherical adsorbate (toxin with molecular weight $M_{m}$ ) molecules of radius $\mathrm{r}$ and diametrical cross-sectional area:

$S_{\mathrm{d}}=\pi r^{2}$

on the surface of spherical granules of magnetically controlled sorbents of radius $R$ with a surface area: $\mathrm{S}_{\mathrm{MYC}}=4 \pi R^{2}$

Suppose that a complex compound (particle or toxin) with molecular weight $\mathrm{M}=\mathrm{m}$ consists of $\mathrm{m}$ identical spherical objects of radius $\mathrm{r} 1$, the so-called elementary objects, and has a spherical shape of radius $\mathrm{rm}$. Let us assume that the radius of a particle of magnetically controlled sorbents is significantly larger than the size of an adsorbate particle. Let's designate the area occupied by an elementary object $S_{1}$. The ratio of the radii $r_{M}$ of a complex compound and an elementary object is determined by the obvious relationship:

$r_{M}=r_{1} \sqrt[3]{m}$

taking into account which we determine the ratio of the areas of their diametrical cross-sections: 


$$
A_{M}=A_{1} \sqrt[3]{m^{2}} \quad\left(\mathrm{~S}_{\mathrm{dm}}=\mathrm{S}_{\mathrm{d} 1} \sqrt[3]{m^{2}}\right) .
$$

Let the maximum permissible concentration (MPC), i.e. the number of molecules per unit volume of the solution containing the

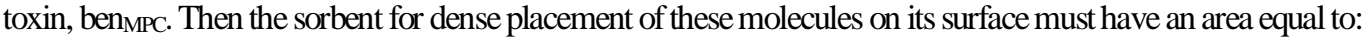

$\mathrm{S}=\mathrm{n}_{\mathrm{MPC} .} \mathrm{S} \mathrm{S}_{\mathrm{d} 1} \sqrt[3]{m^{2}}$,

Accordingly, when working on a linear section of the sorption characteristic, i.e. taking into account the density coefficient, the required sorbent area is determined by the expression:

$\mathrm{S}_{\mathrm{MYC}}=\mathrm{n}_{\mathrm{MPC}} \cdot \mathrm{S}_{\mathrm{dl}} \sqrt[3]{m^{2}} \cdot K_{\mathrm{III}}^{-1}$

The mass of one sorbent granule with density $\rho$, radius $\mathrm{R}$ and volume $\mathrm{V}$ is determined by the formula:

$\mathrm{M}_{\mathrm{MyC}}=\rho \mathrm{V}=\rho \frac{4}{3} \pi R^{3}$

The number of granules of magnetically controlled sorbents in $1 \mathrm{~kg}$ of sorbent is obviously equal to:

$$
N=\frac{1}{\gamma(4 / 3) \pi r_{0}^{3}} \text {. }
$$

Taking into account formula (2), for the surface area of one sorbent granule, we determine the specific surface area, i.e. sorbent sorbent surface of unit weight:

$$
S_{M Y C y d}=\frac{3}{\rho R}
$$

The required mass of the sorbent (in a solution of a unit volume) is determined by the ratio of expressions (3) and (4), from which we obtain the following formula:

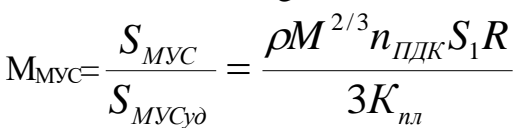

To calculate the required dosage of magnetically controlled sorbents for a specific detoxification procedure, recall that the molecular weights $M_{m}$ of known organic compounds - potential adsorbates are located in a wide range. For example, phenobarbital has a molecular weight, $M_{m}=232$ methylene blue $-M_{m}=319.9$, hemoglobin $-M_{m}=68000$, and the recommended volume of the processed biofluid is usually no more than $50 \mathrm{ml}$.

\section{Conclusion}

The work shows the conditions that ensure the efficiency of the device for extracorporeal detoxification using new technologies using magnetically controlled sorbents. The above technique allows calculating and, accordingly, optimizing the parameters of one of the important components of this device - the unit for sorption and removal of toxins from biofluid. A weighted estimate of the required concentration of magnetically controlled sorbents in the biofluid being purified, the amount and concentration of an emulsion containing a magnetically controlled sorbent, and the duration of the procedure ultimately ensures the safety and effectiveness of this method of medical care.

\section{References}

1. Rabinskiy, L.N., Tushavina, O.V. Problems of land reclamation and heat protection of biological objects against contamination by the aviation and rocket launch site // Journal of Environmental Management and Tourism, 2019, 10(5), p. 967-973.

2. Rabinskiy, L.N., Sitnikov, S.A. Development of technologies for obtaining composite material based on silicone binder for its further use in space electric rocket engines// PeriodicoTcheQuimica, 2018, 15(Special Issue 1), p. 390-395.

3. Astapov, A.N., Kuznetsova, E.L., Rabinskiy, L.N. Operating capacity of anti-oxidizing coating in hypersonic flows of air plasma//Surface Review and Letters, 2019, 26(2), 1850145 p.

4. Rabinskiy, L.N., Tushavina, O.V., Starovoitov, E.I. Study of thermal effects of electromagnetic radiation on the environment from space rocket activity // INCAS Bulletin, 2020, 12(Special Issue), p. 141-148.

5. Babaytsev, A.V., Orekhov, A.A., Rabinskiy, L.N. Properties and microstructure of AlSi10Mg samples obtained by selective laser melting// Nanoscience and Technology, 2020, 11(3), p. 213-222.

6. Bulychev, N.A., Bodryshev, V.V., Rabinskiy, L.N. Analysis of geometric characteristics of two-phase polymer-solvent systems during the separation of solutions according to the intensity of the image of micrographs//PeriodicoTcheQuimica, 2019, 16(32), p. 551-559. 
7. Pronina, P.F., Tushavina, O.V., Starovoitov, E.I. Study of the radiation situation in moscow by investigating elastoplastic bodies in a neutron flux taking into account thermal effects // PeriodicoTcheQuimica, 2020, 17(35), p. 753-764.

8. Dmitriev, V.G., Egorova, O.V., Starovoitov, E.I. Particularities of mathematical modeling of deformation processes for arched and panel designs of composites with large displacements and rotation angles //INCAS Bulletin, 2020, 12(Special Issue), p. 53-66.

9. V.A. Pogodin, L.N. Rabinskii, S.A. Sitnikov. 3D Printing of Components for the Gas-Discharge Chamber of Electric Rocket Engines // Russian Engineering Research. 2019. Vol. 39, No. 9. p. 797-799.

10. Bulychev N. A., Kuznetsova E.L., Bodryshev V. V.Rabinskiy L.N. Nanotechnological aspects of temperature-dependent decomposition of polymer solutions, Nanoscience and Technology: An International Journal, №2, 2018, c.91-97.

11. Formalev, V.F., Kolesnik, S.A., Kuznetsova, E.L. Identification of new law for decomposition of bonding heat-shielding composite materials/Asia Life Sciences. 2019 (1), p. 139-148.

12. Bulychev, N.A., Rabinskiy, L.N., Tushavina, O.V. Effect of intense mechanical vibration of ultrasonic frequency on thermal unstable low-temperature plasma// Nanoscience and Technology, 2020, 11(1), p. $15-21$.

13. A.N. Tarasova. Effect of Vibration on Physical Properties of Polymeric Latexes, International Journal of Pharmaceutical Research, 2020, Vol. 12, Supplementary Issue 2, pp. 1173-1180.

14. B.A. Garibyan. Enhancement of Mechanical Properties of Inorganic Glass under Ultrasonic Treatment, International Journal of Pharmaceutical Research, 2020, Vol. 12, Supplementary Issue 2, pp. 1829-1832.

15. Yu.V. Ioni, A. Ethiraj. Study of Microparticles Surface Modification by Electrokinetic Potential Measuring, International Journal of Pharmaceutical Research, 2020, Vol. 12, Issue 4, pp. 3436-3439.

16. N.A. Bulychev, A.V. Ivanov. Study of Nanostructure of Polymer Adsorption Layers on the Particles Surface of Titanium Dioxide, International Journal of Nanotechnology, 2019, Vol. 16, Nos. 6/7/8/9/10, pp. $356-365$.

17. Yu.V. Ioni. Effect of Ultrasonic Treatment on Properties of Aqueous Dispersions of Inorganic and Organic Particles in Presence of Water-Soluble Polymers, International Journal of Pharmaceutical Research, 2020, Vol. 12, Issue 4, pp. 3440-3442.

18. Egorova, O.V., Kyaw, Y.K. Solution of inverse non-stationary boundary value problems of diffraction of plane pressure wave on convex surfaces based on analytical solution//Journal of Applied Engineering Science, 2020, 18(4), p. 676-680.

19. Rabinskiy, L.N., Tushavina, O.V., Formalev, V.F. Mathematical modeling of heat and mass transfer in shock layer on dimmed bodies at aerodynamic heating of aircraft// Asia Life Sciences, 2019, (2), p. 897911.

20. Antufev, B.A., Egorova, O.V., Rabinskiy, L.N. Quasi-static stability of a ribbed shell interacting with moving load// INCAS Bulletin, 2019, 11, p. 33-39.

21. Bodryshev, V.V., Babaytsev, A.V., Rabinskiy, L.N. Investigation of processes of deformation of plastic materials with the help of digital image processing// PeriodicoTcheQuimica, 2019, 16(33), p. 865-876.

22. A.N. Tarasova. Vibration-based Method for Mechanochemical Coating Metallic Surfaces, International Journal of Pharmaceutical Research, 2020, Vol. 12, Supplementary Issue 2, pp. 1160-1168.

23. A.N. Tarasova. Effect of Reagent Concentrations on Equilibria in Water-Soluble Complexes, International Journal of Pharmaceutical Research, 2020, Vol. 12, Supplementary Issue 2, pp. 1169-1172.

24. B.A. Garibyan. Mechanical Properties of Electroconductive Ceramics, International Journal of Pharmaceutical Research, 2020, Vol. 12, Supplementary Issue 2, pp. 1825-1828.

25. B.A. Garibyan. Modelling of Technical Parameters of Discharge Reactor for Polymer Treatment, International Journal of Pharmaceutical Research, 2020, Vol. 12, Supplementary Issue 2, pp. 1833-1837.

26. M.O. Kaptakov. Effect of Ultrasonic Treatment on Stability of TiO2 Aqueous Dispersions in Presence of Water-Soluble Polymers, International Journal of Pharmaceutical Research, 2020, Vol. 12, Supplementary Issue 2, pp. 1821-1824.

27. Yu.V. Ioni. Synthesis of Metal Oxide Nanoparticles and Formation of Nanostructured Layers on Surfaces under Ultrasonic Vibrations, International Journal of Pharmaceutical Research, 2020, Vol. 12, Issue 4, pp. 3432-3435.

28. N.A. Bulychev, A.V. Ivanov. Effect of vibration on structure and properties of polymeric membranes, International Journal of Nanotechnology, 2019, Vol. 16, Nos. 6/7/8/9/10, pp. $334-343$.

29. N.A. Bulychev, A.V. Ivanov. Nanostructure of Organic-Inorganic Composite Materials Based on Polymer Hydrogels, International Journal of Nanotechnology, 2019, Vol. 16, Nos. 6/7/8/9/10, pp. 344 355.

30. Yu.V. Ioni, A. Ethiraj. New Tailor-Made Polymer Stabilizers for Aqueous Dispersions of Hydrophobic Carbon Nanoparticles, International Journal of Pharmaceutical Research, 2020, Vol. 12, Issue 4, pp. 3443-3446.

31. Yu.V. Ioni. Nanoparticles of noble metals on the surface of graphene flakes, PeriodicoTcheQuimica, 2020, Vol. 17, No. 36, pp. 1199-1211. 
32. Formalev, V.F., Kartashov, É.M., Kolesnik, S.A. On the Dynamics of Motion and Reflection of Temperature Solitons in Wave Heat Transfer in Limited Regions // Journal of Engineering Physics and Thermophysics, 2020, 93(1), p. 10-15.

33. Formalev, V.F., Bulychev, N.A., Kuznetsova, E.L., Kolesnik, S.A. The Thermal State of a Packet of Cooled Microrocket Gas-Dynamic Lasers // Technical Physics Letters, 2020, 46(3), p. 245-248.

34. Formalev, V.F., Kolesnik, S.A., Kuznetsova, E.L., Rabinskiy, L.N. Origination and propagation of temperature solitons with wave heat transfer in the bounded area during additive technological processes // PeriodicoTcheQuimica. 2019. 16(33), p. 505-515.

35. Formalev, V.F., Kolesnik, S.A., Kuznetsova, E.L. Mathematical modeling of a new method of thermal protection based on the injection of special coolants // PeriodicoTcheQuimica. 2019.16(32), p. 598-607.

36. Vakhneev, S., Starovoitov, E. Damping of circular composite viscoelastic plate vibration under neutron irradiation// Journal of Applied Engineering Science, 2020, 18(4), p. 699-704.

37. Formalev, V.F., Kolesnik, S.A., Garibyan, B.A. Mathematical modeling of heat transfer in anisotropic plate with internal sinks // AIP Conference Proceedings, 2019, 2181, 020003.

38. Formalev, V.F., Kolesnik, S.A., Garibyan, B.A. Heat transfer with absorption in anisotropic thermal protection of high-temperature products // Herald of the Bauman Moscow State Technical University, Series Natural Sciences, 2019, (5), p. 35-49.

39. Formalev, V.F., Kolesnik, S.A., Garibyan, B.A. Analytical solution of the problem of conjugate heat transfer between a gasdynamic boundary layer and anisotropic strip //Herald of the Bauman Moscow State Technical University, Series Natural Sciences, 2020, 5(92), p. 44-59.

40. Rabinskiy, L.N., Tushavina, O.V. Composite Heat Shields in Intense Energy Fluxes with Diffusion// Russian Engineering Research, 2019, 39(9), p. 800-803.

41. Rabinskiy, L.N., Tushavina, O.V. Investigation of the influence of thermal and climate effects on the performance of tiled thermal protection of spacecraft//PeriodicoTcheQuimica, 2019, 16(33), p. 657-667. 